\title{
On the boundedness of discrete Wolff potentials
}

\author{
Carme Cascante and Joaquin M. Ortega
}

\begin{abstract}
We obtain characterizations of the pairs of positive measures $\mu$ and $v$ for which the discrete non-linear Wolff-type potential associated to $\mu$ sends $L^{p}(d v)$ into $L^{q}(d \mu)$.
\end{abstract}

Mathematics Subject Classification (2000): 46E30 (primary); 46E35, 31B10 (secondary).

\section{Introduction}

The object of this paper is the study of $L^{p}-L^{q}$ imbeddings of discrete Wolff's potentials assocciated to nonnegative Borel measures.

We recall that Wolff's potentials were introduced originally in [5] in relation to the spectral synthesis problem for Sobolev spaces.

If $\mu$ is a nonnegative Borel measure on $\mathbb{R}^{n}, 1<s<+\infty$ and $\alpha>0$, the Wolff potential associated to $\mu$ is defined by

$$
\mathcal{W}_{\alpha, s}(\mu)(x)=\int_{0}^{+\infty}\left(\frac{\mu(B(x, t))}{t^{n-\alpha}}\right)^{s} \frac{d t}{t}, \quad x \in \mathbb{R}^{n} .
$$

Let $I_{\alpha}(x, y)=\frac{1}{|x-y|^{n-\alpha}}$ be the Riesz kernel in $\mathbb{R}^{n}, 0<\alpha<n$. If $\mu$ is a nonnegative Borel measure on $\mathbb{R}^{n}$, let

$$
I_{\alpha}(\mu)(x)=\int_{\mathbb{R}^{n}} \frac{d \mu(y)}{|x-y|^{n-\alpha}}, x \in \mathbb{R}^{n} .
$$

The nonlinear potential associated to $\mu$ is defined by

$$
V_{\alpha, p}(\mu)(x)=I_{\alpha}\left(\left(I_{\alpha}(\mu)\right)^{p^{\prime}-1} d z\right)(x),
$$

Both authors were partially supported by DGICYT Grant MTM2008-05561-C02-01/MTM, MTM2006-26627-E and DURSI Grant 2005SGR 00611.

Received March 4, 2008; accepted in revised form September 10, 2008. 
and the energy of a positive Borel measure $\mu$ in $\mathbb{R}^{n}$ by

$$
\mathcal{E}_{\alpha, p}(\mu)=\left\|I_{\alpha}(\mu)\right\|_{L^{p^{\prime}(d x)}}^{p^{\prime}}=\int_{\mathbb{R}^{n}} V_{\alpha, p} \mu(x) d \mu(x),
$$

where $\frac{1}{p}+\frac{1}{p^{\prime}}=1$ and the last equality is an immediate consequence of Fubini's theorem. Since there exists a constant $C>0$ such that for any $x \in \mathbb{R}^{n}, \mathcal{W}_{\alpha p, \frac{1}{p-1}}(\mu)(x) \leq$ $C V_{\alpha, p}(\mu)(x)$, we have that $\int_{\mathbb{R}^{n}} \mathcal{W}_{\alpha p, \frac{1}{p-1}}(\mu)(x) d \mu(x) \leq C \mathcal{E}_{\alpha, p}(\mu)$. The converse is the fundamental Wolff's inequality (see [5]), which gives that there exists a constant $C>0$ such that

$$
\mathcal{E}_{\alpha, p}(\mu) \leq C \int_{\mathbb{R}^{n}} \mathcal{W}_{\alpha p, \frac{1}{p-1}}(\mu)(x) d \mu(x) .
$$

Wolff's potentials have applications to many areas of analysis. In the last years, there have been sustantial advances in the solvability of quasilinear and Hessian equations of Lane-Emden type which heavily relies on systematic use of Wolff's potentials, dyadic models and nonlinear trace inequalities (see $[6,7,9]$ and references therein). If $\Omega$ is a bounded domain in $\mathbb{R}^{n}, n \geq 2$, and $\omega$ is a nonnegative Borel locally finite measure on $\Omega$, it is studied in [9] the existence problem for the quasilinear equation

$$
-\operatorname{div} \mathcal{A}(x, \nabla u)=u^{q}+\omega, \quad u \geq 0 \quad \text { in } \quad \Omega, u=0 \quad \text { on } \quad \partial \Omega,
$$

where $p>1, q>p-1$ and $\mathcal{A}(x, \zeta) \cdot \zeta \geq \alpha|\zeta|^{p},|\mathcal{A}(x, \zeta)| \leq \beta|\zeta|^{p-1}$, for some $\alpha, \beta>0$. This equation includes the model problem

$$
-\Delta_{p} u=u^{q}+\omega, \quad u \geq 0 \quad \text { in } \quad \Omega, u=0 \quad \text { on } \quad \partial \Omega,
$$

where $\Delta_{p} u=\operatorname{div}\left(|\nabla u|^{p-2} \nabla u\right)$ is the $p$-Laplacian.

In [9] it has been obtained a criteria for solvability of quasilinear and Hessian equations on the entire space $\mathbb{R}^{n}$, which in particular states:

Theorem 1.1 ([9, Theorem 2.3]). Let $\omega$ be a nonnegative Borel locally finite measure on $\mathbb{R}^{n}, 1<p<n$ and $q>p-1$. Then there exists a nonnegative $\mathcal{A}$ superharmonic solution $u \in L_{\mathrm{loc}}^{q}\left(\mathbb{R}^{n}\right)$ to the equation

$$
-\operatorname{div} \mathcal{A}(x, \nabla u)=u^{q}+\varepsilon \omega, \quad \text { in } \quad \mathbb{R}^{n}, \quad \inf _{x \in \mathbb{R}^{n}} u(x)=0,
$$

for some $\varepsilon>0$ if and only if there is a constant $C>0$ such that

$$
\mathcal{W}_{p, \frac{1}{p-1}}\left(\mathcal{W}_{p, \frac{1}{p-1}}(\omega)\right)^{q}(x) \leq C \mathcal{W}_{p, \frac{1}{p-1}} \omega(x)<+\infty, \quad \text { a.e. }
$$

Moreover, there is a constant $C_{0}$ such that if the above condition holds, with $C \leq$ $C_{0}$, then equation (1.1) has a solution $u$ with $\varepsilon=1$ which satisfies the two-sided pointwise estimate

$$
\frac{1}{K} \mathcal{W}_{p, \frac{1}{p-1}}(\omega)(x) \leq u(x) \leq K \mathcal{W}_{p, \frac{1}{p-1}}(\omega)(x), \quad x \in \mathbb{R}^{n} .
$$


One natural question that arises from the above existence theorem is the study of the following $L^{p}-L^{q}$ trace estimates of the Wolff potential:

Given $0<q<+\infty, 1<p<+\infty$, which are the positive measures $\mu$ on $\mathbb{R}^{n}$ such that

$$
\left\|\left(\mathcal{W}_{p, \frac{1}{p-1}}(f d x)\right)^{p-1}\right\|_{L^{q}(d \mu)} \leq C\|f\|_{L^{p}(d x)},
$$

for any $f \geq 0$ ?

A characterization of such measures would give information on the regularity of the solution of the quasilinear equation given in the above theorem.

We can consider other measures $v$ rather that the Lebesgue measure $d x$, and define the corresponding Wolff type potential: If $s>0, \alpha>0, v$ is a positive Borel measure on $\mathbb{R}^{n}$, and $f$ is a nonnegative $v$-measurable function on $\mathbb{R}^{n}$, let

$$
\mathcal{W}_{\alpha, s}(f d v)(x)=\int_{0}^{+\infty}\left[\frac{\int_{B_{r}(x)} f d \nu}{r^{n-\alpha}}\right]^{s} \frac{d r}{r} .
$$

The general trace problem reads as follows:

Given $0<q, s<+\infty, 1<p<+\infty$, which are the pair of positive Borel measures $\mu, v$ on $\mathbb{R}^{n}$ such that

$$
\left\|\left(\mathcal{W}_{\alpha, s}(f d \nu)\right)^{\frac{1}{s}}\right\|_{L^{q}(d \mu)} \leq C\|f\|_{L^{p}(d v)},
$$

for any $f \geq 0$ ?

In [5] it also was introduced a dyadic version of Wolff's potential. If $\mathcal{D}=\{Q\}$ is the collection of all dyadic cubes in $\mathbb{R}^{n},|Q|$ is the Lebesgue measure of the cube $Q$, and $\mu$ is a positive locally finite Borel measure on $\mathbb{R}^{n}, \mathcal{W}_{\alpha, s}^{\mathcal{D}} \mu$ is defined by

$$
\mathcal{W}_{\alpha, s}^{\mathcal{D}} \mu(x)=\sum_{Q \in \mathcal{D}}\left(\frac{\mu(Q)}{|Q|^{1-\frac{\alpha}{n}}}\right)^{s} \chi_{Q}(x)=\sum_{Q \in \mathcal{D}}\left(\frac{v(Q)}{|Q|^{1-\frac{\alpha}{n}}}\right)^{s}\left(\frac{1}{\mu(Q)} \int_{Q} f d \mu\right)^{s} \chi_{Q}(x) .
$$

Here $\chi_{Q}$ denotes the characteristic function of the cube $Q$. The discrete Riesz potential $I_{\alpha}^{\mathcal{D}}(\mu)$ is

$$
I_{\alpha}^{\mathcal{D}}(\mu)(x)=\sum_{Q \in \mathcal{D}} \frac{\mu(Q)}{|Q|^{1-\frac{\alpha}{n}}} \chi_{Q}(x),
$$

and the discrete energy associated with $\mu$ is given by

$$
\mathcal{E}_{\alpha, p}^{\mathcal{D}}[\mu]=\int_{\mathbb{R}^{n}}\left(I_{\alpha}^{\mathcal{D}}[\mu]\right)^{p^{\prime}} d x=\int_{\mathbb{R}^{n}}\left(\sum_{Q \in \mathcal{D}} \frac{\mu(Q)}{|Q|^{1-\frac{\alpha}{n}}} \chi_{Q}(x)\right)^{p^{\prime}} d x .
$$

An alternative expression for $\mathcal{E}_{\alpha, p}^{\mathcal{D}}$ is an immediate consequence of Fubini's theorem:

$$
\mathcal{E}_{\alpha, p}^{\mathcal{D}}[\mu]=\int_{\mathbb{R}^{n}} I_{\alpha}^{\mathcal{D}}\left[\left(I_{\alpha}^{\mathcal{D}}[\mu]\right)^{p^{\prime}-1} d x\right] d \mu,
$$


where $I_{\alpha}^{\mathcal{D}}\left[\left(I_{\alpha}^{\mathcal{D}}[\mu]\right)^{p^{\prime}-1} d x\right]$ is a dyadic analogue of the nonlinear potential of HavinMaz'ya (see $[1,8])$.

A dyadic version of Wolff's inequality established in [5] shows that, for $1<$ $p<+\infty$, and $v$ a nonnegative Borel measure on $\mathbb{R}^{n}$, there exist constants $C_{1}, C_{2}>$ 0 , such that

$$
C_{1} \mathcal{E}_{\alpha, p}^{\mathcal{D}}[\nu] \leq \int_{\mathbb{R}^{n}} \mathcal{W}_{\alpha, \frac{1}{p-1}}^{\mathcal{D}}[\nu](x) d \nu(x) \leq C_{2} \mathcal{E}_{\alpha, p}^{\mathcal{D}}[\nu]
$$

The purpose of this paper is to study a discrete version of trace estimate (1.3): Given $0<q, s<+\infty, 1<p<+\infty$, which are the pair of positive Borel measures $\mu, v$ on $\mathbb{R}^{n}$ such that

$$
\left\|\left(\mathcal{W}_{\alpha, s}^{\mathcal{D}}(f d \nu)\right)^{\frac{1}{s}}\right\|_{L^{q}(d \mu)} \leq C\|f\|_{L^{p}(d v)}
$$

for any $f \geq 0$ ? The relative position of $p$ and $q$ and of $p$ and $s$ will play an esential role in the proof of the above characterization. The main result we obtain is the following theorem.

Theorem A. Let $0<q, s<+\infty, 1<p<+\infty, 0<\alpha<n$ and $\mu, v$ locally finite positive Borel measures on $\mathbb{R}^{n}$.

1. If $p \leq q$, there exists $C>0$ such that

$$
\left\|\left(\mathcal{W}_{\alpha, s}^{\mathcal{D}}(f d \nu)\right)^{\frac{1}{s}}\right\|_{L^{q}(d \mu)} \leq C\|f\|_{L^{p}(d v)}
$$

if and only if one of the following cases holds:

(i) $s \geq p$ and there exists $C>0$ such that for any $Q \in \mathcal{D}$,

$$
\left(\int_{\mathbb{R}^{n}}\left(\sum_{Q^{\prime} \subset Q}\left(\frac{v\left(Q^{\prime}\right)}{\left|Q^{\prime}\right|^{1-\frac{\alpha}{n}}}\right)^{s} \chi Q^{\prime}\right)^{\frac{q}{s}} d \mu\right)^{\frac{p}{q}} \leq C v(Q) .
$$

(ii) $s<p$ and there exists $C>0$ such that for any cube $Q \in \mathcal{D}$, the following two conditions are satisfied:
(a) $\left(\int_{\mathbb{R}^{n}}\left(\sum_{Q^{\prime} \in \mathcal{D}}\left(\frac{v\left(Q^{\prime}\right)}{\left|Q^{\prime}\right|^{1-\frac{\alpha}{n}}}\right)^{s} \frac{v\left(Q^{\prime} \cap Q\right)}{v\left(Q^{\prime}\right)} \chi Q^{\prime}\right)^{q / s} d \mu\right)^{\frac{1}{(q / s)}} \leq C v(Q)^{s / p}$.
(b) $\left(\int_{\mathbb{R}^{n}}\left(\sum_{Q^{\prime} \in \mathcal{D}}\left(\frac{v\left(Q^{\prime}\right)}{\left|Q^{\prime}\right|^{1-\frac{\alpha}{n}}}\right)^{s} \frac{\mu\left(Q^{\prime} \cap Q\right)}{\mu\left(Q^{\prime}\right)} \chi Q^{\prime}\right)^{(p / s)^{\prime}} d v\right)^{\frac{1}{(p / s)^{\prime}}} \leq C \mu(Q)^{1 /(q / s)^{\prime}}$. 
2. If $q<p$, and the following additional condition is satisfied: There exists $A>0$ such that for any $Q \in \mathcal{D}, x, y \in Q$,

$$
\sum_{Q^{\prime} \subset Q}\left(\frac{v\left(Q^{\prime}\right)}{\left|Q^{\prime}\right|^{1-\frac{\alpha}{n}}}\right)^{s} \chi_{Q^{\prime}}(x) \leq A \sum_{Q^{\prime} \subset Q}\left(\frac{v\left(Q^{\prime}\right)}{\left|Q^{\prime}\right|^{1-\frac{\alpha}{n}}}\right)^{s} \chi_{Q^{\prime}}(y)
$$

where $A$ does not depend on $Q \in \mathcal{D}$. Then there exists $C>0$ such that

$$
\left\|\left(\mathcal{W}_{\alpha, s}^{\mathcal{D}}(f d v)\right)^{\frac{1}{s}}\right\|_{L^{q}(d \mu)} \leq C\|f\|_{L^{p}(d v)},
$$

if and only if one of the following cases hold:

(iii) $s<p$ and

$$
\sum_{Q \in \mathcal{D}}\left(\frac{v(Q)}{|Q|^{1-\frac{\alpha}{n}}}\right)^{s}\left(\frac{\mu(Q)}{v(Q)}\right)^{\frac{p}{p-s}}\left(\sum_{Q^{\prime} \subset Q}\left(\frac{v\left(Q^{\prime}\right)}{\left|Q^{\prime}\right|^{1-\frac{\alpha}{n}}}\right)^{s}\right)^{\frac{p}{p-s}} \chi_{Q} \in L^{\frac{q(p-s)}{s(p-q)}}(d \mu) .
$$

(iv) $p \leq s$ and

$$
\int_{\mathbb{R}^{n}} \sup _{x \in Q}\left(\frac{\left(\sum_{Q^{\prime} \subset Q}\left(\frac{v\left(Q^{\prime}\right)}{\left|Q^{\prime}\right|^{1-\frac{\alpha}{n}}}\right)^{s} \chi_{Q^{\prime}}(x)\right)^{\frac{p}{s}} \mu(Q)}{\nu(Q)}\right)^{\frac{q}{p-q}} d \mu(x)<+\infty .
$$

Observe that in the case that $d v=d x$, the additional condition on the second part of the theorem holds automatically, since for any $Q \in \mathcal{D}$ and $x \in Q$,

$$
\sum_{Q^{\prime} \subset Q}\left(\frac{\left|Q^{\prime}\right|}{\left|Q^{\prime}\right|^{1-\frac{\alpha}{n}}}\right)^{s} \chi_{Q^{\prime}}(x) \simeq|Q|^{\frac{\alpha}{n} s} .
$$

This implies that the original problem (1.2) can be solved without any further hypothesis. In particular, condition (i) corresponding to the case $p \leq q$ and $q<p$ reads as the following simple test condition, namely: there exists $C>0$ such that for any $Q \in \mathcal{D}, \mu(Q) \leq C|Q|^{\frac{q}{p}\left(1-\frac{\alpha p}{n}\right)}$.

Condition (iii) corresponding to the case $q<p$ and $s<p$ reads as

$$
\sum_{Q \in \mathcal{D}}|Q|^{\frac{\alpha s}{n}}\left(\frac{\mu(Q)}{|Q|^{1-\frac{\alpha s}{n}}}\right)^{\frac{p}{p-s}} \chi_{Q} \in L^{\frac{q(p-s)}{s(p-q)}}(d \mu) .
$$


And finally, condition (iv) corresponding to the case $q<p$ and $p \leq s$ is just that

$$
\sup _{x \in Q} \frac{\mu(Q)}{|Q|^{1-\frac{\alpha p}{n}}} \in L^{\frac{q}{p-q}}(d \mu)
$$

In [5] it is shown that there exists $C>0$ such that for any $x \in \mathbb{R}^{n}$,

$$
\mathcal{W}_{\alpha, s}^{\mathcal{D}} v(x)=\sum_{Q \in \mathcal{D}}\left(\frac{v(Q)}{|Q|^{1-\frac{\alpha}{n}}}\right)^{s} \chi_{Q}(x) \leq C \mathcal{W}_{\alpha, s} v(x)=\int_{0}^{+\infty}\left(\frac{\nu(B(x, t))}{t^{n-\alpha}}\right)^{s} \frac{d t}{t}
$$

The results in [5] imply that although the pointwise estimate in the other direction does not hold in general, the $L^{1}(d(v))$-norm of both potentials is equivalent.

In particular from the above pointwise estimate and Theorem $\mathrm{A}$ we deduce necessary conditions, in terms of dyadic cubes, for the continuous general trace problem (1.3).

In [1, page 121], it is introduced a bigger dyadic potential,

$$
\mathcal{U}_{\alpha, s}^{\mathcal{D}} \nu=\sum_{Q \in \mathcal{D}}\left(\frac{v(\widetilde{Q})}{|Q|^{1-\frac{\alpha}{n}}}\right)^{s} \chi_{Q}(x)
$$

which is pointwise equivalent to $\mathcal{W}_{\alpha, s} \nu$. However, the techniques used in this paper for the dyadic potential $\mathcal{W}_{\alpha, s}^{\mathcal{D}} v$ do not apply directly for the bigger potential $\mathcal{U}_{\alpha, s}^{\mathcal{D}} v$ (See Remark 2.10 ).

The proof of Theorem A will be obtained by reformulating it as a particular case of the following problem of discrete multipliers: Given $0<q, s<+\infty$, $1<p<+\infty$, and a sequence $\left(c_{Q}\right)_{Q}$ of nonnegative reals, which are the pair of positive Borel measures $\mu, v$ on $\mathbb{R}^{n}$ such that

$$
\left\|\left(\sum_{Q \in \mathcal{D}} c_{Q^{s}}^{s} \lambda_{Q}^{s} \chi_{Q}\right)^{\frac{1}{s}}\right\|_{L^{q}(d \mu)} \leq C\left\|\sup _{Q \in \mathcal{D}}\left(\lambda_{Q} \chi_{Q}\right)\right\|_{L^{p}(d v)} ?
$$

Notations. Throughout the paper, the letter $C$ may denote various non-negative numerical constants, possibly different in different places. The notation $f(z) \lesssim$ $g(z)$ means that there exists $C>0$, which does not depends of $z, f$ and $g$, such that $f(z) \leq C g(z)$.

ACKnowledgements. We are deeply thankful to Professor Igor Verbitsky for several helpful conversations during the preparation of this paper. 


\section{Discrete multipliers}

We will begin formulating the discrete version of (1.2) we have given in the introduction. The question we want to deal with is then: Given $0<q<+\infty$, $1<p<+\infty$, which are the pair of positive measures $\mu, v$ on $\mathbb{R}^{n}$ such that

$$
\begin{aligned}
& \left\|\left(\sum_{Q \in \mathcal{D}}\left(\frac{v(Q)}{|Q|^{1-\frac{\alpha}{n}}}\right)^{s}\left(\frac{1}{v(Q)} \int_{Q} f d \nu\right)^{s} \chi_{Q}\right)^{\frac{1}{s}}\right\|_{L^{q}(d \mu)}=\left\|\mathcal{W}_{\alpha, s}^{\mathcal{D}}(f d \nu)\right\|_{L^{q}(d \mu)} \\
& \leq C\|f\|_{L^{p}(d v)},
\end{aligned}
$$

for any $f \geq 0$ ?

The following lemma shows that (1.5) can be rewritten in terms of discrete multipliers.

Lemma 2.1. Assume $1<p<+\infty$. Then estimate (1.5) holds if and only if there exists $C>0$ such that for any sequence $\left(\lambda_{Q}\right)_{Q}$ of nonnegative numbers,

$$
\left\|\left(\sum_{Q \in \mathcal{D}}\left(\frac{v(Q)}{|Q|^{1-\frac{\alpha}{n}}}\right)^{s} \lambda_{Q}^{s} \chi_{Q}\right)^{\frac{1}{s}}\right\|_{L^{q}(d \mu)} \leq C\left\|\sup _{Q \in \mathcal{D}}\left(\lambda_{Q} \chi_{Q}\right)\right\|_{L^{p}(d v)} .
$$

Proof. Assume that (1.5) holds, and let $f=\sup _{Q}\left(\lambda_{Q} \chi_{Q}\right)$. Since

$$
\frac{1}{v(Q)} \int_{Q} f d v=\frac{1}{v(Q)} \int_{Q} \sup _{Q^{\prime} \in \mathcal{D}}\left(\lambda_{Q^{\prime}} \chi_{Q^{\prime}}\right) d v \geq \frac{1}{v(Q)} \int_{Q} \lambda_{Q} \chi_{Q} d v=\lambda_{Q},
$$

(1.5) gives that

$$
\left\|\left(\sum_{Q \in \mathcal{D}}\left(\frac{v(Q)}{|Q|^{1-\frac{\alpha}{n}}}\right)^{s} \lambda_{Q}^{s} \chi_{Q}\right)^{\frac{1}{s}}\right\|_{L^{q(d \mu)}} \leq C\left\|\sup _{Q \in \mathcal{D}}\left(\lambda_{Q} \chi_{Q}\right)\right\|_{L^{p}(d v)},
$$

which is (1.2).

Conversely, assume that (1.2) holds, and let $\lambda_{Q}=\frac{1}{v(Q)} \int_{Q} f d \nu, f \geq 0$. We have that since $p>1$, the dyadic maximal operator with respect to $v, M_{v}^{\mathcal{D}}$, given by

$$
M_{v}^{\mathcal{D}} f(x)=\sup _{x \in Q} \frac{1}{v(Q)} \int_{Q} f d v,
$$

is of strong type $(p, p)$ with respect to $v$. Hence the hypothesis gives that

$$
\begin{aligned}
& \left\|\left(\sum_{Q \in \mathcal{D}}\left(\frac{v(Q)}{|Q|^{1-\frac{\alpha}{n}}}\right)^{s}\left(\frac{1}{v(Q)} \int_{Q} f d v\right)^{s} \chi_{Q}\right)^{\frac{1}{s}}\right\|_{L^{q}(d \mu)} \\
& \leq C\left\|\sup _{Q \in \mathcal{D}}\left(\lambda_{Q} \chi_{Q}\right)\right\|_{L^{p}(d v)}=C\left\|M_{v}^{\mathcal{D}} f\right\|_{L^{p}(d v)} \leq C\|f\|_{L^{p}(d v)} .
\end{aligned}
$$


In what follows we will study the more general discrete multiplier problem given by

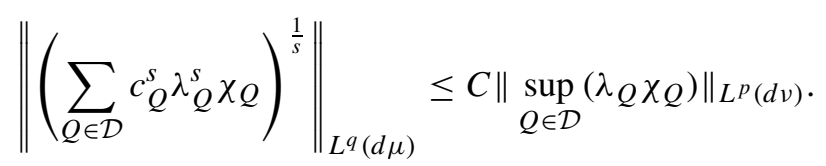

The different characterizations we will obtain depend in the relative position of $p$ and $q$, and in any of the possibilities we will need to consider the different relative positions of $p$ and $s$. More specifically, we will consider the following cases:

(1) $p \leq q$ and $s \geq p$.

(2) $p \leq q$ and $s<p$.

(3) $q<p$ and $s<p$.

(4) $q<p$ and $s \geq p$.

2.1. The case $p \leq q$ and $s \geq p$

If in (1.3) we replace $\lambda_{Q}$ by $\lambda_{Q}^{\frac{1}{p}}$, the estimate can be rewritten in an equivalent way as

$$
\left(\int_{\mathbb{R}^{n}}\left(\sum_{Q \in \mathcal{D}} \lambda_{Q}^{\frac{s}{p}} c_{Q}^{s} \chi_{Q}\right)^{\frac{q}{s}} d \mu\right)^{\frac{p}{q}} \leq C\left\|\sup _{Q \in \mathcal{D}}\left(\lambda_{Q} \chi_{Q}\right)\right\|_{L^{1}(d v)},
$$

where $1 \leq \frac{s}{p}$ and $1 \leq \frac{q}{p}$. Next, this last estimate can be expressed in terms of weighted mixed norms. Namely, if we denote $L_{\mu}^{\frac{q}{p}}\left(l_{c Q}^{\frac{s}{p}}\right)$ the weighted mixed norm space defined by

$$
\begin{aligned}
& L_{\mu}^{\frac{q}{p}}\left(l_{c_{Q}}^{\frac{s}{p}}\right)=\left\{\left(\lambda_{Q} \chi_{Q}\right)_{Q \in \mathcal{D}} ;\left\|\left(\lambda_{Q} \chi_{Q}\right)_{Q \in \mathcal{D}}\right\|_{L_{\mu}^{\frac{q}{p}}\left(l_{c_{Q}}^{\frac{s}{p}}\right)}\right. \\
& \left.=\left(\int_{\mathbb{R}^{n}}\left(\sum_{Q \in \mathcal{D}} \lambda_{Q^{\frac{s}{p}}} c_{Q}^{s} \chi_{Q}\right)^{\frac{q}{s}} d \mu\right)^{\frac{p}{q}}<+\infty\right\},
\end{aligned}
$$

then (1.4) is reformulated as

$$
\left\|\left(\lambda_{Q} \chi_{Q}\right)_{Q \in \mathcal{D}}\right\|_{L_{\mu}^{\frac{q}{p}}\left(l_{c_{Q}}^{\frac{s}{p}}\right)} \leq C\left\|\sup _{Q \in \mathcal{D}}\left(\lambda_{Q} \chi_{Q}\right)\right\|_{L^{1}(d v)} .
$$

Observe that if for any $Q \in \mathcal{D}$ we consider sequences $\left(\lambda Q^{\prime}\right)_{Q^{\prime}}$ satisfying that $\lambda Q^{\prime}=$ 1 for any $Q^{\prime} \subset Q$ and zero elsewhere, we have that

$$
\left\|\sup _{Q^{\prime} \in \mathcal{D}}\left(\lambda_{Q^{\prime}} \chi_{Q^{\prime}}\right)\right\|_{L^{1}(d v)}=v(Q)
$$


and consequently, if (1.5) holds, then

$$
\left\|\left(\chi_{Q^{\prime}}\right)_{Q^{\prime} \in \mathcal{D}}\right\|_{L_{\mu}^{\frac{q}{p}}\left(l_{c}^{\frac{s}{p}}\right)}=\left(\int_{\mathbb{R}^{n}}\left(\sum_{Q^{\prime} \subset Q} c_{Q^{\prime}}^{s} \chi_{Q^{\prime}}\right)^{\frac{q}{s}} d \mu\right)^{\frac{p}{q}} \leq C v(Q) .
$$

The object of the following theorem is to prove that the converse is also true.

Theorem 2.2. If $p \leq q$ and $s \geq p$, the discrete multiplier problem (1.3) (and consequentely (1.5)) holds if and only if there exists $C>0$ such that for any $Q \in \mathcal{D}$,

$$
\left(\int_{\mathbb{R}^{n}}\left(\sum_{Q^{\prime} \subset Q} c_{Q^{\prime}}^{s} \chi_{Q^{\prime}}\right)^{\frac{q}{s}} d \mu\right)^{\frac{p}{q}} \leq C \nu(Q) .
$$

Proof. The necessity of condition (1.7) have just been proved. Before we give the proof of the sufficiency, we make some simplifications:

Step 1. It is enough to show the sufficiency for sequences $\left(\lambda_{Q}\right)_{Q}$ with a finite number of nonzero terms, with constant $C$ which do not depend on the number of nonzero terms. This is a consequence of the Lebesgue monotone convergence theorem.

Step 2. It is enough to show the sufficiency for the case where the finite number of $\lambda Q$ 's different from zero are the ones corresponding to a fixed cube and its descendents. This is due to the fact that if the finite number of nonzero terms correspond to the descendents of $\mathrm{m}$ disjoint cubes $Q_{j} j=1, \cdots, m$, we can deduce this general case from the particular one just observing that

$$
\sup _{Q \in \mathcal{D}}\left(\lambda_{Q} \chi_{Q}\right)=\sup _{Q \subset Q_{1}}\left(\lambda_{Q} \chi_{Q}\right)+\cdots+\sup _{Q \subset Q_{m}}\left(\lambda_{Q} \chi_{Q}\right),
$$

and consequentely,

$$
\begin{aligned}
& \|\left(\lambda_{Q} \chi_{Q}\right)_{Q \in \mathcal{D} \|}{ }_{L_{\mu}^{\frac{q}{p}}\left(l_{c_{Q}}^{\frac{s}{p}}\right)} \\
& \leq\left(\int_{\mathbb{R}^{n}}\left(\sum_{Q^{\prime} \subset Q_{1}} c_{Q^{s}} \chi_{Q^{\prime}}\right)^{\frac{q}{s}} d \mu\right)^{\frac{p}{q}}+\cdots+\left(\int_{\mathbb{R}^{n}}\left(\sum_{Q^{\prime} \subset Q_{m}} c_{Q}^{s} \chi_{Q^{\prime}}\right)^{\frac{q}{s}} d \mu\right)^{\frac{p}{q}} \\
& \leq C\left(\int_{\mathbb{R}^{n}} \sup _{Q \subset Q_{1}}\left(\lambda_{Q} \chi_{Q}\right) d v+\cdots+\int_{\mathbb{R}^{n}} \sup _{Q \subset Q_{m}}\left(\lambda_{Q} \chi_{Q}\right) d \nu\right)=C \int_{\mathbb{R}^{n}} \sup _{Q \in \mathcal{D}}\left(\lambda_{Q} \chi_{Q}\right) d v \\
& =C\left\|\sup _{Q \in \mathcal{D}}\left(\lambda_{Q} \chi_{Q}\right)\right\|_{L^{1}(d v)} .
\end{aligned}
$$


Step 3. By the previous reductions, we just may assume that the finite number of $\lambda$ 's different from zero correspond to a fixed cube $Q^{0}$ and its descendents up to order $m$, which we denote by $Q_{i_{1}, \cdots, i_{k}}^{k}, k=1, \cdots, m i_{1}, \cdots i_{m}=1, \cdots, 2^{n}$. Our next observation is to observe that in addition we may assume that the sequence of $\lambda$ 's satisfies the following monotone condition, namely, $\lambda_{Q^{0}} \leq \lambda_{Q_{i_{1}}^{1}}$ for any $i_{1}=$ $1, \cdots, 2^{n}$, and for any fixed $k=1, \cdots, m$, and $i_{1}, \cdots, i_{k}=1, \cdots, 2^{n}, \lambda_{Q_{i_{1}, \cdots, i_{k}}}^{k} \leq$ $\lambda_{Q_{i_{1}, \cdots, i_{k}, i_{k+1}}^{k+1}}^{k}$ for any $i_{k+1}=1, \cdots, 2^{n}$. Indeed if any of these inequalities does not hold, i.e., there exists $k \in\{1, \cdots, m\}$, and $i_{1}, \cdots i_{k} \in\left\{1, \cdots, 2^{n}\right\}$ with $\lambda_{Q_{i_{1}, \cdots, i_{k}}}^{k}>$

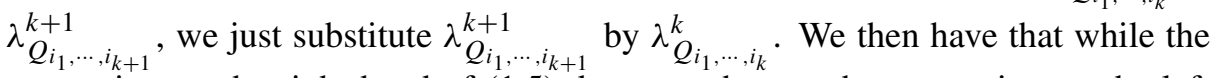
expression on the right hand of (1.5) does not change, the expression on the left hand side increases. We have that for these monotone sequences,

$$
\left\|\sup _{Q \subset Q^{0}}(\lambda Q \chi Q)\right\|_{L^{1}(d v)}=\sum_{i_{1}, \cdots, i_{m}} \lambda_{Q_{i_{1}, \cdots, i_{m}}^{m}}^{m} v\left(Q_{i_{1}, \cdots, i_{m}}^{m}\right) .
$$

In summary, the above steps give that in order to prove the sufficiency it is enough to show the following assertion:

Assume that there exists $C>0$ such that for any $Q^{0}$, and for any fixed finite number of descendents, $Q_{i_{1}, \cdots, i_{k}}^{k}, k=0, \cdots, m, i_{1}, \cdots, i_{m}=1, \cdots, 2^{n}$ (here we are assuming that when $k=0$ we just have the cube $Q^{0}$ ), we have that if $j=0, \cdots, m, i_{1} \cdots, i_{j}=1, \cdots, 2^{n}$,

$$
\left\|\left(\chi_{Q_{i_{1}, \cdots i_{k}}^{k}}\right)_{k=j, \cdots, m ; i_{j+1}, \cdots, i_{m}=1, \cdots, 2^{n}}\right\|_{L_{\mu}^{\frac{q}{p}}\left(l_{c_{Q}}^{\frac{s}{p}}\right)} \leq C v\left(Q_{i_{1}, \cdots, i_{j}}^{j}\right) .
$$

Then for any $\left(\lambda_{Q}\right)_{Q}$, satisfying that $\lambda_{Q} \neq 0$ only if $Q$ is one of the fixed finite number of descendents and satisfying also the monotone condition, we have that if $j=0, \cdots, m, i_{1} \cdots, i_{j}=1, \cdots, 2^{n}$,

$$
\begin{aligned}
& \left\|\left(\lambda_{Q_{i_{1}, \cdots i_{k}}^{k}} \chi_{Q_{i_{1}, \cdots i_{k}}^{k}}\right)_{k=1, \cdots, m ; i_{1}, \cdots, i_{m}=1, \cdots, 2^{n}}\right\|_{L_{\mu}^{\frac{q}{p}}\left(l_{c_{Q}}^{\frac{s}{p}}\right)} \\
& \leq C \sum_{i_{1}, \cdots, i_{m}} \lambda_{Q_{i_{1}, \cdots, i_{m}}^{m}} v\left(Q_{i_{1}, \cdots, i_{m}}^{m}\right)=C\left\|\sup _{Q \subset Q_{0}}\left(\lambda_{Q} \chi_{Q}\right)\right\|_{L^{1}(d v)} .
\end{aligned}
$$

Before we give the proof of the assertion in the above general situation, we begin by briefly sketch the simpler case where the only $\lambda$ 's different from zero correspond to a fixed cube $Q^{0}$, and its first and second generations of descendents, $Q_{i_{1}}^{1}, Q_{i_{1}, i_{2}}^{2}$, $i_{1}, i_{2}=1, \cdots, 2^{n}$. We split the sequence of $\lambda$ 's as a sum of a finite number of 
sequences as follows,

$$
\begin{aligned}
\left(\lambda_{Q} \chi_{Q}\right)_{Q \subset Q^{0}}= & \sum_{i_{1}, i_{2}=1, \cdots, 2^{n}}\left(\left(\lambda_{Q_{i_{1}, i_{2}}^{2}}-\lambda_{Q_{i_{1}}^{1}}\right) \chi_{Q_{i_{1}, i_{2}}^{2}}\right) \\
& +\sum_{i_{1}=1, \cdots, 2^{n}}\left(\left(\lambda_{Q_{i_{1}}^{1}}-\lambda_{Q^{0}}\right) \chi_{Q}\right)_{Q \subset Q_{i_{1}}^{1}} \\
& +\lambda_{Q^{0}}(\chi Q)_{Q \subset Q^{0}} .
\end{aligned}
$$

Since $s / p \geq 1$ and $q / p \geq 1$, the mixed space $L^{\frac{q}{p}}\left(l_{c_{Q}}^{\frac{s}{p}}\right)$ is normed. Then

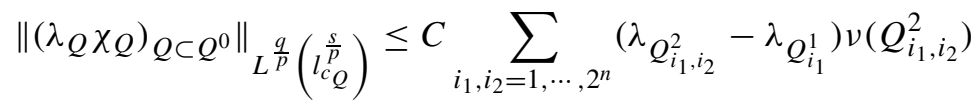

$$
\begin{aligned}
& +\sum_{i_{1}=1, \cdots, 2^{n}}\left(\lambda_{Q_{i_{1}}^{1}}-\lambda_{Q^{0}}\right) v\left(Q_{i_{1}}^{1}\right) \\
& +\lambda_{Q^{0}} v\left(Q^{0}\right) \\
& =C \sum_{i_{1}, i_{2}=1, \cdots, 2^{n}} \lambda_{Q_{i_{1}, i_{2}}^{2}} v\left(Q_{i_{1}, i_{2}}^{2}\right),
\end{aligned}
$$

which gives the desired estimate for this particular situation.

For the general setting, we use the same type of argument and decompose $\left(\lambda_{Q}\right)_{Q}$ as a finite sum of sequences as follows,

$$
\begin{aligned}
& \left(\lambda_{Q} \chi Q\right)_{Q \subset Q^{0}}=\sum_{i_{1}, \cdots, i_{m}=1, \cdots, 2^{n}}\left(\left(\lambda_{Q_{i_{1}, \cdots, i_{m}}^{m}}-\lambda_{Q_{i_{1}, \cdots, i_{m-1}}^{m-1}}\right) \chi_{Q_{i_{1}, \cdots, i_{m}}^{m}}\right)
\end{aligned}
$$

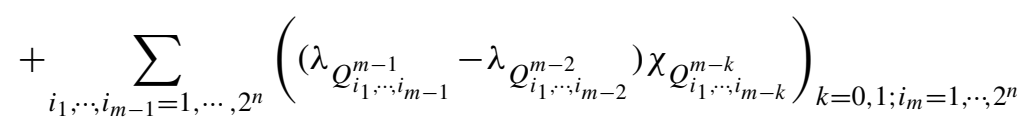

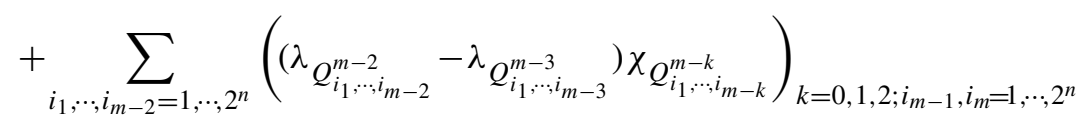

$$
\begin{aligned}
& +\cdots+\lambda Q_{0}(\chi Q)_{Q \subset Q_{0}} .
\end{aligned}
$$

Since by hypothesis the space $L^{\frac{q}{p}}\left(l_{c_{Q}}^{\frac{s}{p}}\right)$ is normed, we obtain from the above de- 
composition that

$$
\begin{aligned}
& \left\|(\lambda Q \chi Q){ }_{Q \subset Q^{0}}\right\|_{L^{\frac{q}{p}}\left(l_{c_{Q}}^{\frac{s}{p}}\right)} \\
& \leq \sum_{i_{1}, \cdots, i_{m}=1, \cdots, 2^{n}}\left(\lambda_{Q_{i_{1}, \cdots, i_{m}}^{m}}-\lambda_{Q_{i_{1}, \cdots, i_{m-1}}^{m-1}}\right)\left\|\left(\chi_{Q_{i_{1}, \cdots, i_{m}}^{m}}\right)\right\|_{L^{\frac{q}{p}}\left(l_{c_{Q}}^{\frac{s}{p}}\right)} \\
& +\sum_{i_{1}, \cdots, i_{m-1}=1, \cdots, 2^{n}}\left(\lambda_{Q_{i_{1}, \cdots, i_{m-1}-1}^{m-1}}-\lambda_{Q_{i_{1}, \cdots, i_{m-2}}^{m-2}}\right)\left\|\left(\chi_{Q_{i_{1}, \cdots, i_{m-k}}^{m-k}}\right)_{\substack{k=0,1 ; \\
i_{m}=1, \cdots, 2^{n}}}\right\|_{L^{\frac{q}{p}}\left(l_{c}^{\frac{s}{p}}\right)} \\
& +\sum_{i_{1}, \cdots, i_{m-2}=1, \cdots, 2^{n}}\left(\lambda_{Q_{i_{1}, \cdots, i_{m-2}}^{m-2}}-\lambda_{Q_{i_{1}, \cdots, i_{m-3}}^{m-3}}\right)\left\|\left(\chi_{Q_{i_{1}, \cdots, i_{m-k}-k}}\right)_{\substack{k=0,1,2 ; \\
i_{m}=1, \cdots, 2^{n}}}\right\|_{L^{\frac{q}{p}}\left(l_{c^{2}}^{\frac{s}{p}}\right)} \\
& +\cdots+\lambda_{Q_{0}}\left\|\left(\chi_{Q}\right)_{Q \subset Q_{0}}\right\|_{L^{\frac{q}{p}}}\left(l_{c_{Q}}^{\frac{s}{p}}\right) .
\end{aligned}
$$

Since (1.8) holds, we have that the above is bounded by

$$
\begin{aligned}
& C\left(\sum_{i_{1}, \cdots, i_{m}=1, \cdots, 2^{n}}\left(\lambda_{Q_{i_{1}, \cdots, i_{m}}^{m}}-\lambda_{Q_{i_{1}, \cdots, i_{m-1}}^{m-1}}\right) v\left(Q_{i_{1}, \cdots, i_{m}}^{m}\right)\right. \\
& +\sum_{i_{1}, \cdots, i_{m-1}=1, \cdots, 2^{n}}\left(\lambda_{Q_{i_{1}, \cdots, i_{m-1}}^{m-1}}-\lambda_{Q_{i_{1}, \cdots, i_{m-2}}^{m-2}}\right) v\left(Q_{i_{1}, \cdots, i_{m-1}}^{m-1}\right) \\
& \left.+\sum_{i_{1}, \cdots, i_{m-2}=1, \cdots, 2^{n}}\left(\lambda_{Q_{i_{1}, \cdots, i_{m-2}}^{m-2}}-\lambda_{Q_{i_{1}, \cdots, i_{m-3}}^{m-3}}\right) v\left(Q_{i_{1}, \cdots, i_{m-2}}^{m-2}\right)+\cdots+\lambda Q_{0} v\left(Q_{0}\right)\right) \\
& =C \sum_{i_{1}, \cdots, i_{m}} \lambda_{Q_{i_{1}, \cdots, i_{m}}^{m}} v\left(Q_{i_{1}, \cdots, i_{m}}^{m}\right)=C\left\|\sup _{Q \subset Q_{0}}\left(\lambda_{Q} \chi_{Q}\right)\right\|_{L^{1}(d v)},
\end{aligned}
$$

which gives (1.3).

\subsection{The case $p \leq q$ and $s<p$}

If we renormalize (1.3) by sustituting $\lambda_{Q}^{s}$ by $\lambda_{Q}$, and denote $\tilde{p}=\frac{p}{s}$ and $\widetilde{q}=\frac{q}{s}$, the estimate can be rewritten in an equivalent way as

$$
\left(\int_{\mathbb{R}^{n}}\left(\sum_{Q \in \mathcal{D}} \lambda_{Q} c_{Q}^{s} \chi Q\right)^{\widetilde{q}} d \mu\right)^{\frac{1}{\widetilde{q}}} \leq C\left\|\sup _{Q \in \mathcal{D}}\left(\lambda Q \chi_{Q}\right)\right\|_{L} \widetilde{p}(d v) .
$$


Lemma 2.1 gives that the above is equivalent to

$$
\left(\int_{\mathbb{R}^{n}}\left(\sum_{Q \in \mathcal{D}} \frac{c_{Q}^{s}}{v(Q)} \chi_{Q} \int_{Q} f d v\right)^{\widetilde{q}} d \mu\right)^{\frac{1}{q}} \leq C\|f\|_{L^{\tilde{p}}(d v)} .
$$

But if we define the discrete operator $T_{\mathcal{D}}$ by

$$
T_{\mathcal{D}}(f d v)=\sum_{Q \in \mathcal{D}} \frac{c_{Q}^{s}}{v(Q)} \chi_{Q} \int_{Q} f d v
$$

the above estimate (1.12) can be rewritten as

$$
\left(\int_{\mathbb{R}^{n}} T_{\mathcal{D}}(f d \nu)^{\tilde{q}}(x) d \mu(x)\right)^{\frac{1}{q}} \leq C\|f\|_{L^{\tilde{p}}(d v)} .
$$

In [10, Theorem 3.2], gives then:

Theorem 2.3 ([10]). If $\widetilde{p} \leq \widetilde{q}$, the estimate (1.13) holds if and only if there exists $C>0$ such that for any $Q \in \mathcal{D}$, the following two conditions are satisfied:

(a) $\left(\int_{\mathbb{R}^{n}} T_{\mathcal{D}}\left(\chi_{Q} d \nu\right)^{\tilde{q}} d \mu\right)^{\frac{1}{q}} \leq C \nu(Q)^{\frac{1}{p}}$.
(b) $\left(\int_{\mathbb{R}^{n}} T_{\mathcal{D}}\left(\chi_{Q} d \mu\right)^{\widetilde{p}^{\prime}} d \nu\right)^{\frac{1}{\widetilde{p}^{\prime}}} \leq C \mu(Q)^{\frac{1}{\widetilde{q}^{\prime}}}$.

As an immediate consequence we have:

Theorem 2.4. If $p \leq q$, and $s<p$, the discrete multiplier problem (1.12) (and consequently (1.3)) holds if and only if there exists $C>0$ such that for any cube $Q \in \mathcal{D}$ the following two conditions are satisfied:

(a) $\left(\int_{\mathbb{R}^{n}}\left(\sum_{Q^{\prime} \in \mathcal{D}} \frac{c_{Q^{\prime}}^{s}}{v\left(Q^{\prime}\right)} v\left(Q^{\prime} \cap Q\right) \chi_{Q^{\prime}}\right)^{\widetilde{q}} d \mu\right)^{\frac{1}{q}} \leq C v(Q)^{\frac{1}{p}}$.
(b) $\left(\int_{\mathbb{R}^{n}}\left(\sum_{Q^{\prime} \in \mathcal{D}} \frac{c_{Q^{\prime}}^{s}}{\mu\left(Q^{\prime}\right)} \mu\left(Q^{\prime} \cap Q\right) \chi_{Q^{\prime}}\right)^{\widetilde{p}^{\prime}} d \nu\right)^{\frac{1}{\widetilde{p}^{\prime}}} \leq C \mu(Q)^{\frac{1}{\widetilde{q}^{\prime}}}$.

In fact, in [10] it is proved that provided we assume some extra mild condition on integrability, in conditions (a) and (b) of Theorem 2.3 it is enough to integrate on the cube $Q$. In particular, if $d v=d x$ and $c_{Q}=\frac{|Q|}{\mid Q^{1-\frac{\alpha}{n}}}$, condition (a) in Theorem 2.4 is reduced to the trivial test condition on cubes: there exists $C>0$ such that for any $Q \in \mathcal{D}, \mu(Q) \leq C|Q|^{\frac{q}{p}\left(1-\frac{\alpha p}{n}\right)}$. 
We observe that the techniques used in the previous subsection, allows to give a simple characterization of (1.3) for the particular case where $p=q, s=1$ and $\mu=v$ which does not use the proof given by [10], and that we think has interest by its own.

Theorem 2.5. Let $1<p<+\infty$, and $\mu$ a positive Borel measure on $\mathbb{R}^{n}$. Then the following assertions are equivalent:

(a) There exists $C>0$ such that for any sequence of nonnegative numbers $\left(\lambda_{Q}\right)_{Q}$,

$$
\left(\int_{\mathbb{R}^{n}}\left(\sum_{Q \in \mathcal{D}} \lambda_{Q} c_{Q} \chi_{Q}\right)^{p} d \mu\right)^{\frac{1}{p}} \leq C\left\|\sup _{Q \in \mathcal{D}}\left(\lambda_{Q} \chi_{Q}\right)\right\|_{L^{p}(d \mu)} .
$$

(b) There exists $C>0$ such that for any sequence of nonnegative numbers $\left(\lambda_{Q}\right)_{Q}$, $\left(\sigma_{Q}\right)_{Q}$,

$$
\sum_{Q \in \mathcal{D}} c_{Q} \mu(Q) \lambda_{Q} \sigma_{Q} \leq C\left(\int_{\mathbb{R}^{n}} \sup _{Q}\left(\lambda_{Q} \chi_{Q}\right)^{p} d \mu\right)^{\frac{1}{p}}\left(\int_{\mathbb{R}^{n} Q} \sup _{Q}\left(\sigma_{Q} \chi_{Q}\right)^{p^{\prime}} d \mu\right)^{\frac{1}{p^{\prime}}}
$$

(c) There exists $C>0$ such that for any $Q \in \mathcal{D}$,

$$
\sum_{Q^{\prime} \subset Q} c_{Q^{\prime}} \mu\left(Q^{\prime}\right) \leq C \mu(Q)
$$

Proof. Duality gives that (a) is equivalent to the discrete bilinear multiplier problem (b). The fact that (b) implies (c) is immediate, if we just consider for any fixed cube $Q \in \mathcal{D}$, the sequence $\left(\lambda_{Q^{\prime}}\right)_{Q^{\prime}}$ such that $\lambda_{Q^{\prime}}=\sigma_{Q^{\prime}}=1$, for any $Q^{\prime} \subset Q$ and zero elsewhere.

If we substitute $c_{Q^{\prime}} \mu\left(Q^{\prime}\right)$ by $c_{Q^{\prime}}$, and use the same reductions of Theorem 2.2 we are left to show the following: Assume that there exists $C>0$ such that for any $Q \in \mathcal{D}$,

$$
\sum_{Q^{\prime} \subset Q} c_{Q^{\prime}} \leq C \mu(Q) .
$$

Then there exists $C>0$ such that for any sequence $\left(\lambda_{Q}\right)_{Q}$ of nonnegative numbers with a finite number of nonzero terms corresponding to a fixed cube $Q^{0}$ and its descendents, and any sequence $\left(\sigma_{Q}\right)_{Q}$ of nonnegative numbers, such that both sequences satisfy the monotone condition given in Step 3, we have

$$
\sum_{Q \in \mathcal{D}} c_{Q^{\lambda}}{ }_{Q} \sigma_{Q} \leq C\left(\int_{\mathbb{R}^{n}} \sup _{Q}\left(\lambda_{Q} \chi_{Q}\right)^{p} d \mu\right)^{\frac{1}{p}}\left(\int_{\mathbb{R}^{n}} \sup _{Q}\left(\sigma_{Q} \chi_{Q}\right)^{p^{\prime}} d \mu\right)^{\frac{1}{p^{\prime}}}
$$

In order to simplify the notations, we will just give the proof for sequences $\left(\lambda Q^{\prime}\right){ }_{Q^{\prime}}$, $\left(\sigma_{Q^{\prime}}\right)_{Q^{\prime}}$, with nonzero terms corresponding to a cube, and its first and second generation of decendents, which we will denote by $Q^{0}, Q_{i_{1}}, i_{1}=1, \cdots, 2^{n}$ and $Q_{i_{1}, i_{2}}$, 
$i_{1}, i_{2}=1, \cdots, 2^{n}$, respectively, and satisfying the monotone condition. We have that (1.17) gives that the following estimates are satisfied:

$$
\begin{aligned}
& \sum_{i_{1}, i_{2}=1, \cdots, 2^{n}} c_{Q_{i_{1}, i_{2}}}+\sum_{i_{1}=1, \cdots, 2^{n}} c_{Q_{i_{1}}}+c_{Q^{0}} \leq C \sum_{i_{1}, i_{2}} \mu\left(Q_{i_{1}, i_{2}}\right)=\mu\left(Q^{0}\right), \\
& \sum_{i_{2}=1, \cdots, 2^{n}} c_{Q_{i_{1}, i_{2}}}+c_{Q_{i_{1}}} \leq C \mu\left(Q_{i_{1}}\right), \quad i_{1}=1, \cdots, 2^{n} \\
& c_{Q_{i_{1}, i_{2}}} \leq C \mu\left(Q_{i_{1}, i_{2}}\right), \quad i_{1}, i_{2}=1, \cdots, 2^{n} .
\end{aligned}
$$

We have that

$$
\begin{aligned}
& \sum_{i_{1}, i_{2}=1, \cdots, 2^{n}} c_{Q_{i_{1}, i_{2}}} \lambda_{Q_{i_{1}, i_{2}}} \sigma_{Q_{i_{1}, i_{2}}}+\sum_{i_{1}=1, \cdots, 2^{n}} c_{Q i_{1}} \lambda_{Q_{i_{1}}} \sigma_{Q_{i_{1}}}+c_{Q^{0}} \lambda_{Q^{0}} \sigma_{Q^{0}} \\
& =\sum_{i_{1}=1, \cdots, 2^{n}} \sum_{i_{1}=1, \cdots, 2^{n}}\left(\lambda Q_{i_{1}, i_{2}} \sigma_{Q_{i_{1}, i_{2}}}-\lambda Q_{Q_{i_{1}}} \sigma_{Q_{i_{1}}}\right) c_{Q_{i_{1}, i_{2}}} \\
& +\sum_{i_{1}=1, \cdots, 2^{n}}\left(\lambda_{Q_{i_{1}}} \sigma_{Q_{i_{1}}}-\lambda_{Q^{0}} \sigma_{Q^{0}}\right)\left(c_{Q_{i_{1}}}+\sum_{i_{2}=1, \cdots, 2^{n}} c_{Q_{i_{1}, i_{2}}}\right) \\
& +\lambda{ }_{Q^{0}} \sigma_{Q^{0}}\left(\sum_{i_{1}, i_{2}=1, \cdots, 2^{n}} c_{Q_{i_{1}, i_{2}}}+\sum_{i_{1}=1, \cdots, 2^{n}} c_{Q_{i_{1}}}+c_{Q^{0}}\right) \\
& \leq C \sum_{i_{1}=1, \cdots, 2^{n}} \sum_{i_{2}=1, \cdots, 2^{n}}\left(\lambda_{Q_{i_{1}, i_{2}}} \sigma_{Q_{i_{1}, i_{2}}}-\lambda_{Q_{i_{1}}} \sigma_{Q_{i_{1}}}\right) \mu\left(Q_{i_{1}, i_{2}}\right) \\
& +\sum_{i_{1}=1, \cdots, 2^{n}}\left(\lambda_{Q_{i_{1}}} \sigma_{Q_{i_{1}}}-\lambda_{Q^{0}} \sigma_{Q^{0}}\right)\left(\sum_{i_{2}=1, \cdots, 2^{n}} \mu\left(Q_{i_{1}, i_{2}}\right)\right) \\
& +\lambda Q_{Q^{0}} \sigma_{Q^{0}}\left(\sum_{i_{1}, i_{2}=1, \cdots, 2^{n}} \mu\left(Q_{i_{1}, i_{2}}\right)\right)=C \sum_{i_{1}, i_{2}=1, \cdots, 2^{n}} \lambda Q_{Q_{i_{1}, i_{2}}} \sigma_{Q_{i_{1}, i_{2}}} \mu\left(Q_{i_{1}, i_{2}}\right) \\
& \leq C\left(\sum_{i_{1}, i_{2}=1, \cdots, 2^{n}} \lambda_{Q_{i_{1}, i_{2}}^{p}}^{p} \mu\left(Q_{i_{1}, i_{2}}\right)\right)^{\frac{1}{p}}\left(\sum_{i_{1}, i_{2}=1, \cdots, 2^{n}} \sigma_{Q_{i_{1}, i_{2}}^{p^{\prime}}}^{p^{\prime}} \mu\left(Q_{i_{1}, i_{2}}\right)\right)^{\frac{1}{p^{\prime}}} .
\end{aligned}
$$

The general case is proved anagously to Theorem 2.2.

The version of the above theorem for general pairs of measures $\mu$ and $\nu$ does not hold in general. The condition (c) which corresponds to the general case is now given by: There exists $C>0$ such that for any $Q \in \mathcal{D}$,

$$
\sum_{Q^{\prime} \subset Q} c_{Q^{\prime}} \mu\left(Q^{\prime}\right) \leq C \mu(Q)^{\frac{1}{p}} \nu(Q)^{\frac{1}{p^{\prime}}} .
$$

The following example gives that (1.19) is not, in general, sufficient in order that the discrete bilinear problem holds. 
Proposition 2.6. There exists a pair of positive measures $\mu \neq v$ on $\mathbb{R}^{n}$ and a sequence $\left(c_{Q}\right)_{Q}$ of nonnegative numbers satisfying condition (1.19) with $C=1$ but where the estimate

$$
\sum_{Q \in \mathcal{D}} c_{Q} \mu(Q) \lambda_{Q} \sigma_{Q} \leq\left(\int_{\mathbb{R}^{n}} \sup _{Q}\left(\lambda_{Q} \chi_{Q}\right)^{2} d \mu\right)^{\frac{1}{2}}\left(\int_{\mathbb{R}^{n}} \sup _{Q}\left(\sigma_{Q} \chi_{Q}\right)^{2} d v\right)^{\frac{1}{2}}
$$

does not hold for every sequences $\left(\lambda_{Q}\right)_{Q},\left(\sigma_{Q}\right)_{Q}$ of nonnegative numbers.

Proof. We fix a cube $Q^{0} \in \mathcal{D}$, and consider the first generation of its descendents, that we denote by $Q_{i}, i=1, \cdots, 2^{n}$. We will construct a sequence of non negative numbers $\left(c_{Q}\right)_{Q}$ satisfying that $c_{P}=0$ for any cube $P$ different from $Q^{0}$ and its first generation of descendents. In such situation, condition (1.19) reduces to

$$
\begin{aligned}
& \sum_{i} c_{Q_{i}} \mu\left(Q_{i}\right)+c_{Q^{0}} \mu\left(Q^{0}\right) \leq \mu\left(Q^{0}\right)^{\frac{1}{2}} \nu\left(Q^{0}\right)^{\frac{1}{2}}, \\
& c_{Q_{i}} \mu\left(Q_{i}\right) \leq \mu\left(Q_{i}\right)^{\frac{1}{2}} \nu\left(Q_{i}\right)^{\frac{1}{2}}, \quad i=1, \cdots, 2^{n} .
\end{aligned}
$$

On the other hand, estimate (1.20) for nonnegative numbers satisfying $\lambda Q_{i} \geq \lambda Q_{Q^{0}}$, $\sigma_{Q_{i}} \geq \sigma_{Q^{0}}, i=1, \cdots, 2^{n}$, can be rewritten as

$$
\begin{aligned}
& \sum_{i} \lambda_{Q_{i}} \sigma_{Q_{i}} c_{Q_{i}} \mu\left(Q_{i}\right)+\lambda_{Q^{0}} \sigma_{Q^{0}} c_{Q^{0}} \mu\left(Q^{0}\right) \\
& \leq\left(\sum_{i} \lambda_{Q_{i}}^{2} \mu\left(Q_{i}\right)\right)^{\frac{1}{2}}\left(\sum_{i} \sigma_{Q_{i}}^{2} \nu\left(Q_{i}\right)\right)^{\frac{1}{2}} .
\end{aligned}
$$

We define the sequence $\left(c_{Q}\right)_{Q}$ in terms of the measures $\mu$ and $v$ (to be constructed) as follows:

$$
\begin{aligned}
& c_{Q_{i}} \mu\left(Q_{i}\right)=v\left(Q_{i}\right)^{\frac{1}{2}} \mu\left(Q_{i}\right)^{\frac{1}{2}}, \quad i=1, \cdots, 2^{n} \\
& c_{Q} \mu\left(Q^{0}\right)=\mu\left(Q^{0}\right)^{\frac{1}{2}} \nu\left(Q^{0}\right)^{\frac{1}{2}}-\sum_{i} \nu\left(Q_{i}\right)^{\frac{1}{2}} \mu\left(Q_{i}\right)^{\frac{1}{2}} .
\end{aligned}
$$

Observe that by Hölder's inequality, $c_{Q} \geq 0$.

With that choice, and for the particular case where $\lambda_{Q^{0}}=\sigma_{Q^{0}}=1,(1.21)$ reduces to

$$
\begin{aligned}
& \sum_{i} \lambda_{Q_{i}} \sigma_{Q_{i}} \nu\left(Q_{i}\right)^{\frac{1}{2}} \mu\left(Q_{i}\right)^{\frac{1}{2}}+\left(\mu\left(Q^{0}\right)^{\frac{1}{2}} \mu\left(Q^{0}\right)^{\frac{1}{2}}-\sum_{i} \mu\left(Q_{i}\right)^{\frac{1}{2}} \nu\left(Q_{i}\right)^{\frac{1}{2}}\right) \\
& \leq\left(\sum_{i} \lambda_{Q_{i}}^{2} \mu\left(Q_{i}\right)\right)^{\frac{1}{2}}\left(\sum_{i} \sigma_{Q_{i}}^{2} \nu\left(Q_{i}\right)\right)^{\frac{1}{2}}
\end{aligned}
$$


for any $\lambda_{Q_{i}} \geq 1, \sigma_{Q_{i}} \geq 1, i=1, \cdots, 2^{n}$. But the above can be written as

$$
\begin{aligned}
& \left(\sum_{i} v\left(Q_{i}\right)\right)^{\frac{1}{2}}\left(\sum_{i} \mu\left(Q_{i}\right)\right)^{\frac{1}{2}}-\sum_{i} v\left(Q_{i}\right)^{\frac{1}{2}} \mu\left(Q_{i}\right)^{\frac{1}{2}} \\
& \leq\left(\sum_{i} \lambda_{Q_{i}}^{2} v\left(Q_{i}\right)\right)^{\frac{1}{2}}\left(\sum_{i} \mu_{Q_{i}}^{2} \mu\left(Q_{i}\right)\right)^{\frac{1}{2}}-\sum_{i} \lambda_{Q_{i}} \sigma_{Q_{i}} v\left(Q_{i}\right)^{\frac{1}{2}} \mu\left(Q_{i}\right)^{\frac{1}{2}} .
\end{aligned}
$$

If we consider the vectors in $\mathbb{R}^{2^{n}}$ given by

$$
\begin{array}{ll}
u^{v}=\left(v\left(Q_{1}\right)^{\frac{1}{2}}, \cdots, v\left(Q_{2^{n}}\right)^{\frac{1}{2}}\right), & u^{\mu}=\left(\mu\left(Q_{1}\right)^{\frac{1}{2}}, \cdots, \mu\left(Q_{2^{n}}\right)^{\frac{1}{2}}\right) \\
v_{\lambda}^{v}=\left(\lambda_{Q_{1}} v\left(Q_{1}\right)^{\frac{1}{2}}, \cdots, \lambda_{Q_{2^{n}}} v\left(Q_{2^{n}}\right)^{\frac{1}{2}}\right), & v_{\sigma}^{\mu}=\left(\sigma_{Q_{1}} \mu\left(Q_{1}\right)^{\frac{1}{2}}, \cdots, \sigma_{Q_{2^{n}}} \mu\left(Q_{2^{n}}\right)^{\frac{1}{2}}\right),
\end{array}
$$

this last inequality reduces to

$$
\left\|u^{v}\right\|_{2}\left\|v^{\mu}\right\|_{2}-u^{v} \cdot v^{\mu} \leq\left\|u_{\lambda}^{\nu}\right\|_{2}\left\|v_{\sigma}^{\mu}\right\|_{2}-u_{\lambda}^{v} \cdot v_{\sigma}^{\mu},
$$

for any $\lambda_{Q_{i}} \geq 1, \sigma_{Q_{i}} \geq 1, i=1, \cdots, 2^{n}$. Now, we just need to define the measures $v$ and $\mu$ such that the vectors $u^{v}$ and $v^{\sigma}$ are close to be "orthogonal", and $\lambda_{Q_{i}} \geq 1$, $\sigma_{Q_{i}} \geq 1, i=1, \cdots, 2^{n}$, such that the vectors $u_{\lambda}^{\nu}$ and $v_{\sigma}^{\mu}$ are equals to finish with the construction. For instance, if $0<\varepsilon<1$, consider

$$
\left.\left.u^{\mu}=(1, \varepsilon, \cdots, 1, \varepsilon)\right), \quad u^{v}=(\varepsilon, 1, \cdots, \varepsilon, 1)\right)
$$

and $\lambda_{Q_{2 k+1}}=1, \lambda_{Q_{2 k}}=\frac{1}{\varepsilon}, k=0, \cdots, 2^{n-1}-1, \sigma_{Q_{2 k+1}}=\frac{1}{\varepsilon}, \sigma_{Q_{2 k}}=1, k=$ $0, \cdots, 2^{n-1}-1$. Then

$$
\left\|u^{v}\right\|_{2}\left\|v^{\mu}\right\|_{2}-u^{v} \cdot v^{\mu}=2^{\frac{(n-1)}{2}}\left(1+\varepsilon^{2}\right)^{\frac{1}{2}}-2^{n} \varepsilon,
$$

whereas $\left\|u_{\lambda}^{v}\right\|_{2}\left\|v_{\sigma}^{\mu}\right\|_{2}-u_{\lambda}^{v} \cdot v_{\sigma}^{\mu}=0$.

\subsection{The case $q<p$ and $s<p$}

As in the case $p \leq q$ and $s<p$, if in (1.3) we substitute $\lambda_{Q}^{s}$ by $\lambda_{Q}$, put $\tilde{p}=\frac{p}{s}$ and $\widetilde{q}=\frac{q}{s}$, and we obtain that the estimate can be rewritten as

$$
\left(\int_{\mathbb{R}^{n}}\left(\sum_{Q \in \mathcal{D}} \lambda_{Q} c_{Q}^{s} \chi_{Q}\right)^{\widetilde{q}} d \mu\right)^{\frac{1}{\tilde{q}}} \leq C\left\|\sup _{Q \in \mathcal{D}}\left(\lambda_{Q} \chi_{Q}\right)\right\|_{L} \tilde{p}(d \nu),
$$

where now $0<\widetilde{q}<\tilde{p}$ and $\tilde{p}>1$. Using again Lemma 2.1 we have that the above is equivalent to

$$
\left(\int_{\mathbb{R}^{n}}\left(\sum_{Q \in \mathcal{D}} \frac{1}{|Q|_{\nu}} \int_{Q} f d \nu c_{Q}^{s} \chi Q\right)^{\widetilde{q}} d \mu\right)^{\frac{1}{\widetilde{q}}} \leq C\|f\|_{L} \tilde{p}(d v) .
$$


This inequality has been studied in [4]. In order to write down the characterization, we need to introduce some more notations.

If $K: \mathcal{D} \rightarrow \mathbb{R}^{+}$, and $v$ is a positive Borel measure on $\mathbb{R}^{n}$, we define the generalizad Riesz dyadic operator $T_{K}^{\mathcal{D}}$ given by

$$
T_{K}^{\mathcal{D}}[v]=\sum_{Q \in \mathcal{D}} v(Q) K(Q) \chi_{Q}
$$

We also define the function $\bar{K}(Q)(x)$ supported on $Q$ given by

$$
\bar{K}(Q)(x)=\frac{1}{|Q|_{\nu}} \sum_{Q^{\prime} \subset Q} K\left(Q^{\prime}\right) v\left(Q^{\prime}\right) \chi_{Q^{\prime}}(x) .
$$

We say that the pair $(K, v)$ satisfies the so-called dyadic logarithmic bounded oscillation condition (DLBO):

$$
\sup _{x \in Q} \bar{K}(Q)(x) \leq A \inf _{x \in Q} \bar{K}(Q)(x),
$$

where $A$ does not depend on $Q \in \mathcal{D}$. Assume $K$ is a radially nonincreasing kernel and $d v=d x$ or $K(Q)=r_{Q}^{n-\alpha}, 0<\alpha<n$ and $v$ satisfies a dyadic reverse condition, i.e. there exists $C>0$ and $\gamma>n-\alpha$ such that for any $j \geq 0, Q \in \mathcal{D}$, $v\left(2^{j} Q\right) \geq C 2^{j \gamma} v(Q)$, where $2^{j} Q$ is the unique dyadic cube in $\mathcal{D}$ such that $Q \subset$ $2^{j} Q$ and $r_{2^{j} Q}=2^{j} r_{Q}$. Then in any of these cases we obtain that the pair $(K, v)$ satisfies the (DLBO) condition (see [3] for more details).

For $(K, v) \in(\mathrm{DLBO})$, we set $\bar{K}(Q)=\inf _{x \in Q} \bar{K}(Q)(x), Q \in \mathcal{D}$, if $v(Q) \neq 0$, and $\bar{K}(Q)=0$ if $v(Q)=0$. The generalized Wolff potential of a measure $\sigma$ introduced in [3] can be defined when the pair $(K, v)$ satisfies the DLBO condition in an equivalent way by:

$$
\mathcal{W}_{K, \nu}^{\mathcal{D}}[\sigma](x)=\sum_{Q \in \mathcal{D}} K(Q)[\bar{K}(Q)]^{p^{\prime}-1}[\sigma(Q)]^{p^{\prime}-1} v(Q) \chi_{Q}(x) .
$$

Theorem 2.7 ([4, Theorem 2.1]). Let $K: \mathcal{D} \rightarrow \mathbb{R}^{+}, 0<q<p<+\infty$, and $1<p<+\infty$. Let $\mu$ and $\sigma$ be nonnegative Borel measures on $\mathbb{R}^{n}$. Suppose that $(K, v) \in(\mathrm{DLBO})$. Then there exists a constant $C>0$ such that the trace inequality

$$
\int_{\mathbb{R}^{n}}\left|T_{K_{\mathcal{D}}}[f d \nu]\right|^{q} d \mu \leq C\|f\|_{L^{p}(d v)}^{q}, \quad f \in L^{p}(d v),
$$

holds if and only if

$$
\mathcal{W}_{K, \nu}^{\mathcal{D}}[\mu] \in L^{\frac{q(p-1)}{p-q}}(d \mu)
$$


Given $\left(c_{Q}\right)_{Q}$ a sequence of nonnegative real numbers, we define

$$
\bar{C}_{s}(Q)(x)=\sum_{Q^{\prime} \subset Q} c_{Q^{\prime}}^{s} \chi_{Q^{\prime}}(x) .
$$

We will say that the pair $\left(\left(c_{Q}^{s}\right)_{Q}, v\right)$ satisfies the DLBO condition if the pair $(K, v)$ satisfies the DLBO condition, where $K(Q)=\frac{c_{Q}^{s}}{\nu(Q)}$. In that case we define $\bar{C}_{Q, s}=$ $\inf _{x \in Q} \bar{C}_{s}(Q)(x)$, which by hypothesis is equivalent to $\sup _{x \in Q} \bar{C}_{s}(Q)(x)$. The Wolff-type potential is now

$$
\mathcal{W}_{K, v}^{\mathcal{D}}[\sigma](x)=\sum_{Q \in \mathcal{D}} c_{Q}^{s}\left[\bar{C}_{Q, s}\right]^{(p / s)^{\prime}-1}\left(\frac{\mu(Q)}{v(Q)}\right)^{(p / s)^{\prime}-1} \chi_{Q}(x) .
$$

We can now state the characterization.

Theorem 2.8. Let $1<p<+\infty, q<p$ and $s<p$, and let $\mu$, $v$ be two nonnegative Borel measures on $\mathbb{R}^{n}$. Assume that the pair $\left(\left(c_{Q}^{s}\right)_{Q}\right.$, v) satisfies the DLBO condition. We then have that (1.22) (and consequently (1.3)) holds if and only if:

$$
\sum_{Q \in \mathcal{D}} c_{Q}^{s}\left(\frac{\mu(Q)}{v(Q)}\right)^{\frac{p}{p-s}} \bar{C}_{Q, s}^{\frac{p}{p-s}} \chi_{Q} \in L^{\frac{q(p-s)}{s(p-q)}}(d \mu) .
$$

2.4. The case $q<p$ and $p \leq s$

With the same substitution of the previous case, we have that the estimate (1.3) can be rewritten as

$$
\left(\int_{\mathbb{R}^{n}}\left(\sum_{Q \in \mathcal{D}} \lambda_{Q} c_{Q}^{s} \chi_{Q}\right)^{\tilde{q}} d \mu\right)^{\frac{1}{q}} \leq C\left\|\sup _{Q \in \mathcal{D}}\left(\lambda_{Q} \chi_{Q}\right)\right\|_{L^{\tilde{p}}(d v)},
$$

where now $0<\widetilde{q}<\widetilde{p} \leq 1$.

Theorem 2.9. Let $q<p$ and $p \leq s$, and let $\mu, v$ be two nonnegative Borel measures on $\mathbb{R}^{n}$. Assume that the pair $\left(\left(c_{Q}^{s}\right)_{Q}, v\right)$ satisfies the DLBO condition. We then have that (1.28) (and consequently (1.3)) holds if and only if:

$$
\int_{\mathbb{R}^{n} x \in Q} \sup _{x \in Q}\left(\frac{\left(\sum_{Q^{\prime} \subset Q} c_{Q^{\prime}}^{s} \chi_{Q^{\prime}}\right)^{\frac{p}{s}} \mu(Q)}{v(Q)}\right)^{\frac{q}{p-q}} d \mu<+\infty .
$$


Proof. We begin with the proof of the necessity. If $Q \in \mathcal{D}$, take $\lambda_{Q}=\sum_{Q \subset Q^{\prime}} \rho_{Q^{\prime}}$. Since $\widetilde{p} \leq 1$, we have that

$$
\left(\sum_{Q \subset Q^{\prime}} \rho_{Q^{\prime}}\right)^{\tilde{p}} \leq\left(\sum_{Q^{\prime} \in \mathcal{D}} \rho_{Q^{\prime}}\right)^{\tilde{p}} \leq \sum_{Q^{\prime} \in \mathcal{D}} \rho_{Q^{\prime}}^{\widetilde{p}}
$$

On the other hand,

$$
\sum_{Q \in \mathcal{D}} \lambda_{Q} c_{Q}^{s} \chi_{Q}=\sum_{Q \in \mathcal{D}} c_{Q}^{s} \sum_{Q \subset Q^{\prime}} \rho_{Q^{\prime}} \chi_{Q}=\sum_{Q^{\prime} \in \mathcal{D}} \rho_{Q^{\prime}} \sum_{Q \subset Q^{\prime}} c_{Q}^{s} \chi_{Q} .
$$

Consequently if (1.28) is satisfied, we obtain that

$$
\begin{aligned}
& \left(\int_{\mathbb{R}^{n}}\left(\sum_{Q^{\prime} \in \mathcal{D}} \rho_{Q^{\prime}} \sum_{Q \subset Q^{\prime}} c_{Q}^{s} \chi Q\right)^{\tilde{q}} d \mu\right)^{\frac{1}{\tilde{q}}}=\left(\int_{\mathbb{R}^{n}}\left(\lambda_{Q} c_{Q}^{s} \chi_{Q}\right)^{\tilde{q}} d \mu\right)^{\frac{1}{\tilde{q}}} \\
& \leq C\left\|\sup _{Q \in \mathcal{D}}\left(\lambda_{Q} \chi_{Q}\right)\right\|_{L^{\tilde{p}}(d v)}=C\left\|\sup _{Q \in \mathcal{D}}\left(\sum_{Q \subset Q^{\prime}} \rho_{Q^{\prime}} \chi_{Q}\right)\right\|_{L^{\widetilde{p}}(d v)} \\
& =C\left\|\sup _{Q \in \mathcal{D}}\left(\sum_{Q \subset Q^{\prime}} \rho_{Q^{\prime}} \chi Q\right)^{\tilde{p}}\right\|_{L^{1}(d v)}^{\frac{1}{p}} \leq C\left\|\sum_{Q^{\prime} \in \mathcal{D}} \rho_{Q^{\prime}}^{\widetilde{p}} \chi_{Q^{\prime}}\right\|_{L^{1}(d v)}^{\frac{1}{p}} \\
& =\left(\sum_{Q^{\prime} \in \mathcal{D}} \rho_{Q^{\prime}}^{\tilde{p}} v\left(Q^{\prime}\right)\right)^{\frac{1}{\tilde{p}}} \text {. }
\end{aligned}
$$

So we have shown that if (1.28) holds, then

$$
\left(\int_{\mathbb{R}^{n}}\left(\sum_{Q^{\prime} \in \mathcal{D}} \rho_{Q^{\prime}} \bar{C}_{Q, s} \chi Q\right)^{\tilde{q}} d \mu\right)^{\frac{1}{\tilde{q}}} \leq C\left(\sum_{Q^{\prime} \in \mathcal{D}} \rho_{Q^{\prime}}^{\tilde{p}} \nu\left(Q^{\prime}\right)\right)^{\frac{1}{\tilde{p}}}
$$

Applying the characterization in [11, Theorem 3.d] (since $\widetilde{q}<\tilde{p} \leq 1$ ), we have that the above holds if and only if

$$
\int_{\mathbb{R}^{n}} \sup _{x \in Q}\left(\frac{\left(\bar{C}_{Q, s} \chi Q\right)^{\frac{p}{s}} \mu(Q)}{v(Q)}\right)^{\frac{q}{p-q}} d \mu<+\infty
$$

which is what we wanted to prove. 
Conversely, we have that if we apply Hölder's inequality with exponent $\frac{\widetilde{p}}{q}>1$, we obtain:

$$
\begin{aligned}
& \int_{\mathbb{R}^{n}}\left(\sum_{Q \in \mathcal{D}} \lambda_{Q} c_{Q}^{s} \chi_{Q}\right)^{\tilde{q}} d \mu \\
& \leq\left(\int_{\mathbb{R}^{n}}\left(\sum_{Q \in \mathcal{D}} \lambda_{Q} c_{Q}^{s} \chi_{Q}(x)\right)^{\tilde{p}} \frac{d \mu(x)}{\sup _{x \in Q} \bar{C}_{Q, s}^{\tilde{p}} \frac{\mu(Q)}{\nu(Q)}}\right)^{\frac{\tilde{q}}{p}} \\
& \quad \times\left(\int_{\mathbb{R}^{n}}\left(\sup _{x \in Q} \bar{C}_{Q, s} \frac{\mu(Q)}{v(Q)}\right)^{\frac{\tilde{p}}{p-q}-1} d \mu(x)\right)^{\frac{\tilde{p}-\tilde{q}}{\tilde{p}}} .
\end{aligned}
$$

The second term on the right is finite since we are assuming that (1.29) holds. For the estimate of the first term on the right, we will use that by Theorem 2.2,

$$
\begin{aligned}
& \left(\int_{\mathbb{R}^{n}}\left(\sum_{Q \in \mathcal{D}} \lambda_{Q} c_{Q}^{s} \chi_{Q}(x)\right)^{\tilde{p}} \frac{d \mu(x)}{\sup _{x \in Q} \bar{C}_{Q, s}^{\tilde{p}} \frac{\mu(Q)}{v(Q)}}\right)^{\frac{1}{p}} \\
& \leq C\left\|\sup _{Q \in \mathcal{D}}\left(\lambda_{Q} \chi_{Q}\right)\right\|_{L^{\tilde{p}}(d v)},
\end{aligned}
$$

if and only if

$$
\sup _{Q \in \mathcal{D}} \frac{1}{v(Q)} \int_{Q}\left(\sum_{Q^{\prime} \subset Q} c_{Q^{\prime}}^{s} \chi Q_{Q^{\prime}}(x)\right)^{\tilde{p}} \frac{d \mu(x)}{\sup _{x \in Q^{\prime}} \bar{C}_{Q^{\prime}, s}^{\tilde{p}} \frac{\mu\left(Q^{\prime}\right)}{v\left(Q^{\prime}\right)}}<+\infty .
$$

But

$$
\int_{Q}\left(\sum_{Q^{\prime} \subset Q} c_{Q^{\prime}}^{s} \chi Q^{\prime}(x)\right)^{\tilde{p}} \frac{d \mu(x)}{\sup _{x \in Q} \bar{C}_{Q, s}^{\tilde{p}} \frac{\mu(Q)}{v(Q)}} \leq \int_{Q}\left(\bar{C}_{Q, s} \chi_{Q}\right)^{\tilde{p}} \frac{d \mu(x)}{\bar{C}_{Q, s}^{\tilde{p}} \frac{\mu(Q)}{v(Q)}}=v(Q),
$$

and hence (1.31) holds. Plugging this in (1.30), we deduce that

$$
\int_{\mathbb{R}^{n}}\left(\sum_{Q \in \mathcal{D}} \lambda_{Q} c_{Q}^{s} \chi_{Q}\right)^{\tilde{q}} d \mu \leq\left\|\sup _{Q \in \mathcal{D}}\left(\lambda_{Q} \chi_{Q}\right)\right\|_{L \tilde{p}(d v)}^{\widetilde{q}},
$$

and that finishes the proof of the theorem. 
Remark 2.10. It is worthwhile to mention a pointwise estimate that relates the continuous Wolff potential $\mathcal{W}_{\alpha, s} v$ considered in this paper and another type of discrete potential. If $\widetilde{Q}=3 Q$, for any $Q \in \mathcal{D}$, we denote by

$$
\mathcal{U}_{\alpha, s}^{\mathcal{D}} v(x)=\sum_{Q \in \mathcal{D}}\left(\frac{v(\widetilde{Q})}{|Q|^{1-\frac{\alpha}{n}}}\right)^{s} \chi_{Q}(x)
$$

Following the arguments of the estimate in [1, (4.7.15)], it can be shown that there exists $C>0$ such that for any $x \in \mathbb{R}^{n}$,

$$
\frac{1}{C} \mathcal{W}_{\alpha, s} v(x) \leq \mathcal{U}_{\alpha, s}^{\mathcal{D}} v(x) \leq C \mathcal{W}_{\alpha, s} v(x)
$$

Indeed, if $x \in \mathbb{R}^{n}$ and $k \in \mathbb{Z}$, let $Q_{k} \in \mathcal{D}_{\widetilde{Q}}$ be the unique dyadic cube such that $x \in Q_{k}$ and $\left|Q_{k}\right|=2^{n k}$. Then $B\left(x, 2^{k}\right) \subset \widetilde{Q}_{k}$, and consequently,

$$
\begin{aligned}
& \mathcal{W}_{\alpha, s} v(x)=\int_{0}^{+\infty}\left(\frac{\nu(B(x, t))}{t^{n-\alpha}}\right)^{s} \frac{d t}{t} \\
& \preceq \sum_{k \in \mathbb{Z}}\left(\frac{\nu\left(B\left(x, 2^{k}\right)\right)}{2^{k(n-\alpha)}}\right)^{s} \preceq \sum_{k \in \mathbb{Z}}\left(\frac{v\left(\widetilde{Q}_{k}\right)}{\left|Q_{k}\right|^{1-\frac{\alpha}{n}}}\right)^{s} \chi Q_{k}(x)=C \mathcal{U}_{\alpha, s}^{\mathcal{D}} \nu(x) .
\end{aligned}
$$

In addition, if we fix a nonnegative integer $m$ such that $2^{-m+1} \sqrt{n} \leq 1$, then if $x \in Q_{k},\left|Q_{k}\right|=2^{n k}$, we have that $\widetilde{Q}_{k} \subset B\left(x, 2^{m+k}\right)$, and thus

$$
\begin{aligned}
\mathcal{U}_{\alpha, s}^{\mathcal{D}} \nu(x) & \leq \sum_{k \in \mathbb{Z}}\left(\frac{v\left(B\left(x, 2^{m+k}\right)\right)}{2^{k(n-\alpha)}}\right)^{s} \\
& \preceq \sum_{k \in \mathbb{Z}}\left(\frac{\nu\left(B\left(x, 2^{k}\right)\right)}{2^{k(n-\alpha)}}\right)^{s} \simeq \mathcal{W}_{\alpha, s} v(x) .
\end{aligned}
$$

Observe that if $v$ is a doubling measure, i.e., there exists $C>0$ such that for any $x \in \mathbb{R}^{n}, R>0, v(B(x, 2 R)) \leq C v(B(x, R))$, we have that the two discrete potentials $\mathcal{U}_{\alpha, s}^{\mathcal{D}} v$ and $\mathcal{W}_{\alpha, s}^{\mathcal{D}} v$ above introduced satisfy that there exists $A>0$ such that for any $x \in \mathbb{R}^{n}$,

$$
\frac{1}{A} \mathcal{W}_{\alpha, s}^{\mathcal{D}} v(x) \leq \mathcal{U}_{\alpha, s}^{\mathcal{D}} v(x) \leq C \mathcal{W}_{\alpha, s}^{\mathcal{D}} v(x)
$$

But since the measure $f d v$ which appears in (1.3) is not doubling for all $f$, the above equivalence can not be used to obtain an equivalent to theorem $\mathrm{A}$ for the continuous general trace problem.

It would be interesting to characterize the inequality (1.5) for the discrete potential $\mathcal{U}_{\alpha, s}^{\mathcal{D}} \nu$. However, the methods applied in this paper for the discrete potential $\mathcal{W}_{\alpha, s}^{\mathcal{D}} v$ can not directly be used for the bigger discrete potential $\mathcal{U}_{\alpha, s}^{\mathcal{D}} v$. 


\section{References}

[1] D. R. Adams and L. I. Hedberg, "Function Spaces and Potential Theory", SpringerVerlag, Berlin-Heidelberg-New York, 1996.

[2] C. Cascante, J. M. Ortega and I. E. Verbitsky, Trace inequalities of Sobolev type in the upper triangle case, Proc. London Math. Soc. 80 (2000), 391-414.

[3] C. Cascante, J. M. Ortega and I. E. Verbitsky, Nonlinear potentials and two weight trace inequalities for general dyadic and radial kernels, Indiana Univ. Math. J. 53 (2004), $845-882$.

[4] C. Cascante, J. M. Ortega and I. E. Verbitsky, On $L^{p}-L^{q}$ inequalities, J. London Math. Soc. 7 (2006), 497-511.

[5] L. I. Hedberg and Th. H. WolfF, Thin sets in nonlinear potential theory, Ann. Inst. Fourier (Grenoble) 33 (1983), 161-187.

[6] T. KILPLELAÏNEN and J. MALÝI, The Wiener test and potential estimates for quasilinear elliptic equations, Acta Math. 172 (1994), 137-161.

[7] D. A. LABUtin, Potential estimates for a class of fully nonlinear elliptic equations, Duke Math. J. 111 (2002), 1-48.

[8] V. G. MAZ'YA, “Sobolev Spaces”, Springer-Verlag, Berlin-Heidelberg-New York, 1985.

[9] N. C. PhUC and I. E. Verbitsky, Quasilinear and Hessian equations of Lane-Emden type, Comm. Partial Differential Equations 31 (2006), 1779-1791.

[10] E. T. SAWYER, R. L. WHEEDEN and S. ZHAO, Weighted norm inequalities for operators of potential type and fractional maximal functions, Potential Anal. 5 (1996), 523-580.

[11] I. E. VERBITSKY, Imbedding and multiplier theorems for discrete Littlewood-Paley spaces, Pacific J. Math. 176 (1996), 529-556.

[12] I. E. VERBITSKY, Nonlinear potentials and trace inequalities, Oper. Theory Adv. Appl. 110 (1999), 323-343.

Departament de Matemàtica Aplicada i Anàlisi Facultat de Matemàtiques

Universitat de Barcelona

Gran Via 585

08071 Barcelona, Spain

cascante@ub.edu

ortega@ub.edu 